\title{
DEVELOPMENTS IN LEGAL CHEMISTRY. SIBUTRAMINE EFFECTS THE BOUNDARY BETWEEN LEGAL AND ILLEGAL CONSUMPTION
}

\author{
D.M. Pancu
}

\author{
Diana Mihaela Pancu \\ Student at the master's program "Applied Forensic Research", \\ Police Academy "Alexandru Ioan Cuza", Bucharest, Romania \\ * Correspondence: Diana Mihaela Pancu, Police Academy "Alexandru Ioan Cuza" Bucharest, \\ 1-3 Aleea Privighetorilor St., Sector 1, 014031Bucharest, Romania \\ E-mail:dianapancu@yahoo.com
}

\begin{abstract}
In the present paper we determined the presence of sibutramine in urine using the GCMS/MS system. The determination of sibutramine was studied in relation to slimming health foods ${ }^{l}$. Prolonged or excessive consumption of unauthorized pharmaceuticals may cause serious adverse consequences on health. In this study, samples were extracted with the help of methanol and acetonitryl and the sibutramine concentrations were found in the range of $0,5 \mathrm{~g} / \mathrm{l}$.
\end{abstract}

Keywords: sibutramine, GC-MS/MS, methanol and acetonitryl, HPLC-MS

\section{Introduction}

Amphetamines are psychostimulant drugs that produce increased wakefulness, decreased fatigue and appetite.

The pressor effect of amphetamine was first described by Piness and associates (1933). In 1933, it was noted that it is a bronchodilator, a respiratory stimulant with analeptic action and was compared to epinephrine. Amphetamines described as methamphetamine and dextroamphetamine belongs to the group of drugs that potentially increase the levels of norepinephrine, serotonin and dopamine, euphoria-inducing drugs absorbed at cellular level.

From a medical perspective, amphetamines are sympathomimetic substances, related to derivatives of adrenaline (epinephrine) in which central excitatory effects prevail. Amphetamine is a stimulant of the nervous system due to its weak and long-term pressor action (approximately ten times longer than that of adrenaline).

Amphetamine was first synthesized in 1887 by the Romanian Lazar Edeleanu in Berlin, Germany. The name derives from phenylisopropylamine. It was one of a series of compounds obtained from plants ephedrine was derived from, that had been isolated by Ma Huang together with Nagayoshi Nagai.

Adrenaline and noradrenaline are hormones secreted by the adrenal glands, located in the upper pole of the kidney, and more specifically, the renal medulla. Both hormones exert similar effects of sympathetic stimulation, but while norepinephrine has more intense vascular actions, adrenaline activates especially the energy metabolism.

Amphetamine activity at the brain level is specific; certain receptors that respond to amphetamine in several brain regions do not conduct the impulse to other regions, although they exert effects on behaviour by carrying neurotransmitters in the brain, including

\footnotetext{
${ }^{11}$ Yamamoto S-Shokuhin Eiseigaku Zasshi, 369, 2011.
} 
dopamine, serotonin and norepinephrine. So dopamine $D_{2}$ receptors in the hippocampus - the brain region associated with memory formation - appear not to be affected by the presence of amphetamines.

\section{I.1. CHEMICHAL STRUCTURE}

In terms of illicit production, alongside amphetamine, the following are obtained:

- Specific chemical (the chemical used as green material, precursor, reagent or solvent in illegal processes or substance control).

- Precursor (chemicals which in clandestine processes are incorporated, in full or in part, in the final production of the molecule of the substance under international control).

-Reagent (chemical that reacts or participates in the reaction, but does not become part of the final product).

- Solvent (is the liquid substance that dissolves another solid substance without changing the chemical composition, and does not become part of the final product).

- Impurities (natural constituents originating from plant materials or from materials obtained through the processing of those remaining in the final product after complete process conversion).

- Adulterants (pharmacologically active substances remaining or added after the conversion of the final product). volume).

- Diluent (pharmacologically inactive substance added to the final product to increase

Amphetamines belong to the class of phenylalkylamines and classify into:

Amphetamines (found under the following names: 1-phenylpropan-2-amine,

$\square$ methyl-benzeneethanamine

$\square$ methylphenethylamine)

Methamphetamine (found under the following names:

2-(methylamino)-1-phenyl-1-propanone

$\mathrm{N}, \square$ dimethylaminobenzene

N, $\square$. dimethylphenethyl-benzene

N-methyl-amphethamine

Phenylisopropylmethylamine)

Sibutramine (found under the following names: Meridia, Ectiva, Reductil,

1-[1-(4-chlorophenyl) cyclo-butyl]-NH,3-trimethyl-butane-1-amine)

Methylphenidate (found under the following names: centedrin, ritalin)

Amfepramone (found under the name: Diethylpropion amphetamine)

Fenfluramine (found under the name: fenfluramine)

\section{I.2. SYNTHESIS}

Amphetamine is prepared from benzyl-methyl-ketone, by using several methods commonly used to convert the carbonyl group into the group $-\mathrm{CH}_{2} \mathrm{NH}_{2}$ (reduction of the nitrogen functional derivatives of carbonyl compounds). Benzyl-methyl-ketone is obtained by heating a mixture of phenylacetic acid and acetic acid at $400 \mathrm{C}$, in the presence of thorium dioxide or through the Friedel - Crafts reaction between benzene and chloroacetone, in the presence of aluminum chloride. 


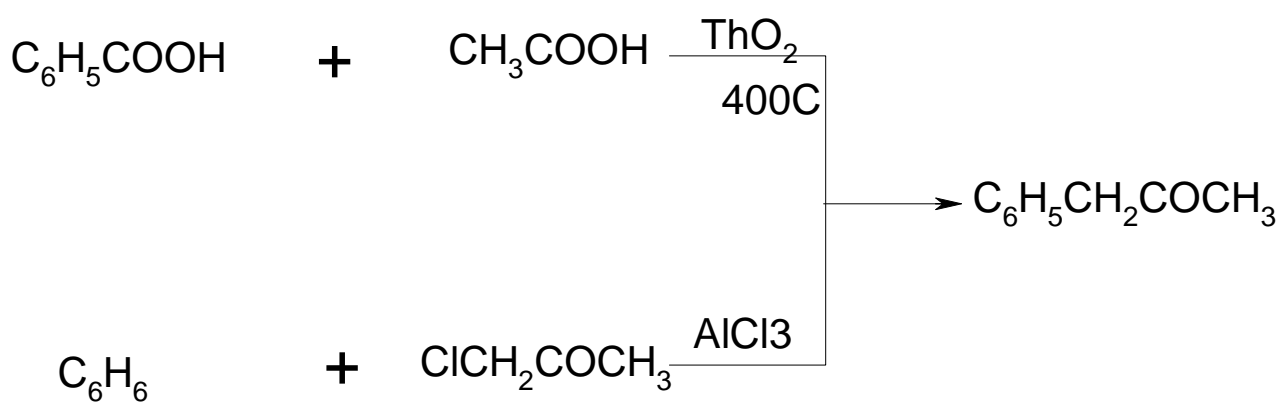

\section{I.3. FORMULATION}

Amphetamine-products generally come in the form of sulfates or phosphates. They are available on the international market in the form of tablets, capsules, syrups and elixirs. The vast majority of amphetamines appear as hydrochlorides or sulfates.

In terms of solubility, amphetamines are insoluble in water. As free bases they are soluble in organic solvents such as ethanol, diethyl ether, and chloroform. Hydrochlorides are soluble in water and ethanol, slightly soluble in chloroform and insoluble in diethyl ether. Sulphates and phosphates are soluble in water, slightly soluble in ethanol and insoluble in diethyl ether and chloroform. Trimethylamphetamine have boiling points ranging between 118 C - 220 C.

Amphetamine has two optical isomers:

- Dextroamphetamine - dextrorotatory stereoisomer

- Levoamphetamine - levorotary stereoisomer

Dexamphetamine is 2-4 times more active than the racemic compound as a psychomotor stimulant and less active as sympathomimetic. Also the dextrorotatory isomer is 24 times more active than its levorotary enantiomer.

Methamphetamine has amphetamine-like properties but its sympathomimetic effects are weak in normal doses.

Methylphenidate is a chemical related to amphetamines, having weaker psychomotor stimulant properties and weak peripheral actions. The effect is fast and relatively short in duration, corresponding to a half-life of 1-2 hours, which is an advantage over amphetamine.

Fenfluramine is a halogenated amphetamine derivative. Amphetamines may react with $\mathrm{H}_{3} \mathrm{PO}_{4}$ and $\mathrm{H}_{2} \mathrm{SO}_{4}$ forming amphetamine sulphate and phosphate. Amphetamine derivatives are:

A. Dimethoxy-amphetamines -2.5-dimethoxy-amphetamine (DMA)

-4bromo-2.5- dimethoxy-amphetamine (DOB)

- 2.5-dimethoxy-4-methylamphetamine (STP, DOM)

- 3.4 - methylenedioxyamphetamine

- 1-methoxy-4-amphetamine

- dimethoxy-2, 5-ethyl-4-amphetamine

- methoxy-3-methylenedioxy-4,5-amphetamine

B. Trimethoxy-amphetamines - 3,4,5-trimethoxyamphetamine (TMA 1)

- 2,4,5-trimethoxyamphetamine (TMA 2)

- 2,3,4-trimethox yamphetamine (TMA 3)

- 2,3,5-trimethoxyamphetamine (TMA 4)

- 2,3,4-trimethoxyphenyl-propan-2-amine

- 2,3,6-trimethoxyamphetamine (TMA 5)

- 2,4,6- trimethoxyphenyl-propane (TMA 6) 


\section{I.4 CHEMICHAL DETERMINATION}

The most common methods for the determination of amphetamines in biological products are spectrophotometric, immunologic fluorescence - FPIA (Fluorescence Polarisation Imunoassay), immunochromatographic, gas or liquid chromatography. These include:

I.5.1. The spectrophotometric method - in this case, amphetamine engage, in alkaline medium, with diazotized p-nitroaniline resulting in a calorimetric red azo-derivative.

I.5.2. The immunofluorescence method - is used in screening tests for testing a group of subjects suspected of amphetamine or methamphetamine consumption, using urine as a bioassay. For an amount of urine of $150 \mu \mathrm{l} /$ determination, results are obtained in a period of 12 to 14 minutes. Results are given as present or absent, because of the methods implemented using a six point-calibration curve, rather than a 1-point calibration for the FPIA assay system - the cut-off value being used for the determination of xenobiotic substances in the urine by reading the light polarization vector in polarization milliunits. A positive result will be necessarily confirmed by the GC-MS method.

I.5.3. Immunochromatographic method - is the appearance of a coloured band on a solid support (one to check, another to validate the presence or absence of amphetamines or methamphetamines in the urine, saliva or sweat, the type of biological fluids depending on the manufacturer of the immunochromatographic test). It also uses urine to detect: opiates, cocaine, phencyclidine, cannabis, barbiturates, benzodiazepines, tricyclic antidepressants. A positive result will be necessarily confirmed by the GC-MS method.

I.5.4. The gas-chromatographic method coupled with the GC-MS mass spectrophotometer

I.5.5. The HPLC-MS - DAD method

II. EXPERIMENTAL

\section{II.1 Materials} used:

To determine the amphetamines- sibutramine in this case the following materials were

- Spectrophotometer Cary 5 - Varian

- FPIA AXSYM - Abbot system

- GC-MS/MS Saturn 2000 - Varian system

- HPLC-MS/MS-DAD system - Varian

- Eppendorf centrifuge 5616

- Analytical balance - Precisa 40SM-200A

- Magnetic stirrer - HP 240

- Ultrasonic bath - Branson 2210

- Thermostat - Memmert

- Accessories and reagents specific to an analytical toxicology laboratory

- Sibutramine (10 mg amphetamine - Zentiva)

Sibutramine (trade name Meridia in the U.S. and Canada, Ectiva in South Africa, Reductil in Europe and most other countries), is an orally administered agent for the treatment of obesity as an appetite suppressant. It has virtually no potential for abuse because of the lack of dopaminergic effects. It is used as an anorexic, which is the only reason for its classification as a controlled drug, as in the mid-20th century it resulted in a number of cases of abuse or addiction. 


\section{II.2. METHODS}

II.2.1. Gas chromatography method coupled with the GC-MS mass spectrometer

It consists of three submethods for each device that is part of the GC-MS system. Thus there will be a method for autosampler 8200, one for gas chromatograph 3800 and one for mass spectrometer Saturn 2000.

Autosampler 8200

Number of containers - 48; Volume injection syringe - $10 \mu 1$

Number of liquids for washing injection syringe -2

Time for washing injection syringe with a washer fluid $-20 \mathrm{~s}$

Time of taking over the injection matrix - between air plugs

Injection volume $-1 \mu \mathrm{l}$; Depth of penetration of the needle into the bottle $-80 \%$

Fluid intake speed $-1 \mu \mathrm{l} / \mathrm{s}$; Heating time for the needle in the injector- $6 \mathrm{~s}$

Injection speed $-10 \mu \mathrm{l} / \mathrm{s}$; Time of the needle remaining in the injector after injection- $6 \mathrm{~s}$

\section{Gas chromatography 3800}

Injector type 1079; Injection temperature $-300^{\circ} \mathrm{C}$

Split rate $5 \%$ constant for the duration of the analysis; Constant flow $-1.2 \mathrm{ml} / \mathrm{min}$

Oven temperature program, $\mathrm{T}_{0}-180^{\circ} \mathrm{C}$ waiting time $1.1 \mathrm{~min}$

Growth in $\mathrm{T} 1-290^{\circ} \mathrm{C}$ with $5^{\circ} \mathrm{C} / \mathrm{min}$ speed; $\mathrm{T} 1-290^{\circ} \mathrm{C}$ waiting time $-13.9 \mathrm{~min}$

Saturn 2000

Filament off - 0 - 3min, AGC field "full scan" - 50 - $400 \mathrm{amu}$; Time 3 - $35 \mathrm{~min}$

Scan duration - 1s/scan; Filament current - $10 \mu \mathrm{A}$; Maximum number of ions 25,000 Ionization maximum duration $25 \mathrm{~ms}$; Prescan duration $100 \mu \mathrm{s}$

Mass to lower fund $-45 \mathrm{amu}$

\section{RESULTS}

In the conditions mentioned, sibutramine extracted by two methods from a capsule of $10 \mathrm{mg}$ Amphetamine - Zentiva was highlighted.

The extraction was performed from $7.5 \mathrm{mg}$ excipient in methanol and acetonytril 1: 1 and $7.5 \mathrm{mg}$ excipient in dichloroethane, dichloromethane and chloroform 1: 1: 1 . The solutions were ultrasonated for one hour and centrifuged for 10 minutes. The supernatant was the injection matrix for both the gas chromatographic and the HPLC methods. Fig. 1 shows the resulting total ion chromatogram following the injection of sibutramine solution with a concentration of $0.5 \mathrm{mg} / \mathrm{l}$. 


\section{Chromatogram Plot}

File: |lsaturn2000\hdd saturn2lsaturnldatalprb24664.ms

Sample: URINA 6/16/09 1:19 PM

Sample Notes: SIBUTRAMINE (EXTRACTIE IN TEI SOLVENTI)

Operator: MIHAI Scan Range: 1 - 3000 Time Range: 0.01 - $50.00 \mathrm{~min}$.

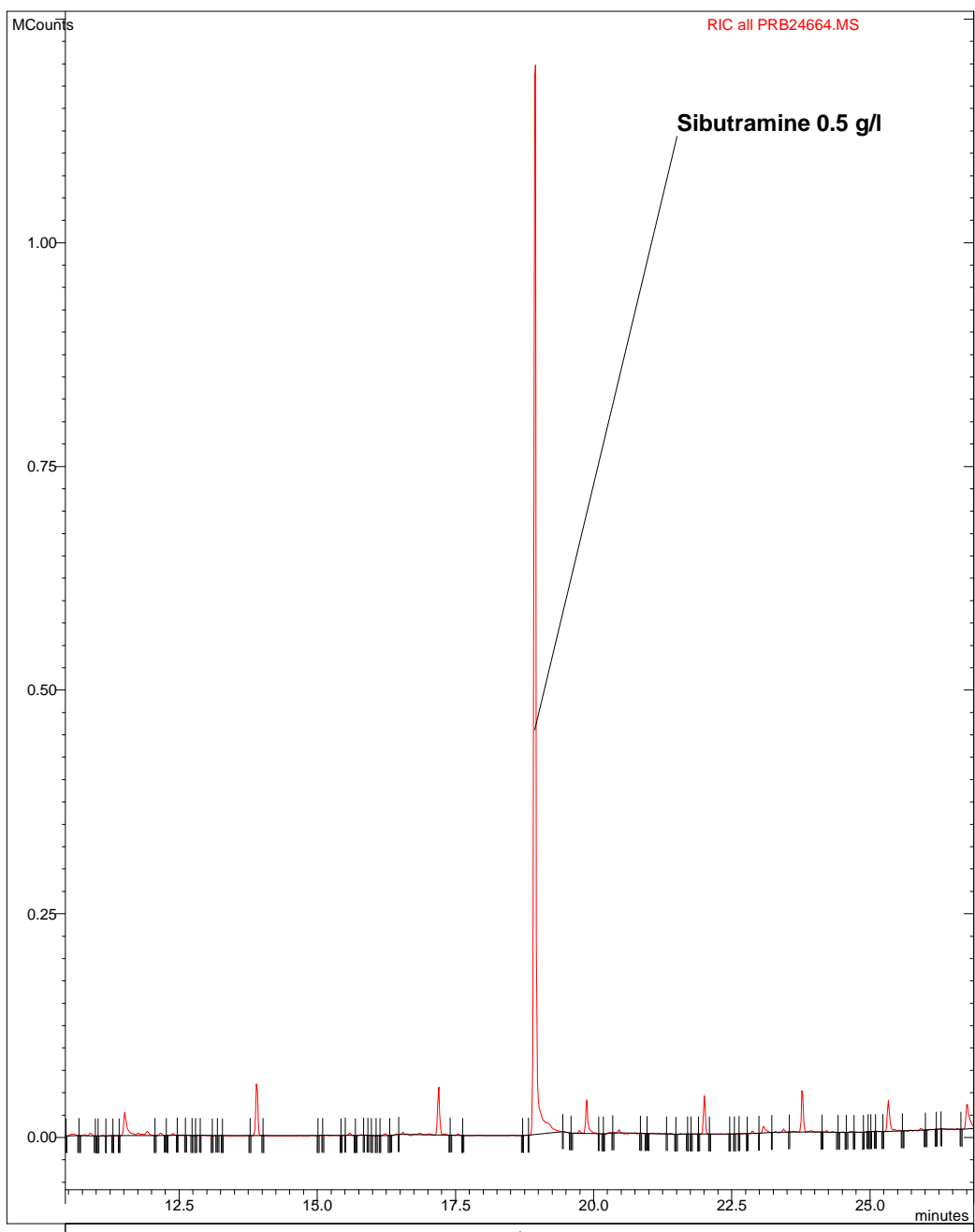

Fig. 1. Total ion chromatogram of sibutramine at a concentration of $0.5 \mathrm{~g} / \mathrm{l}$. 

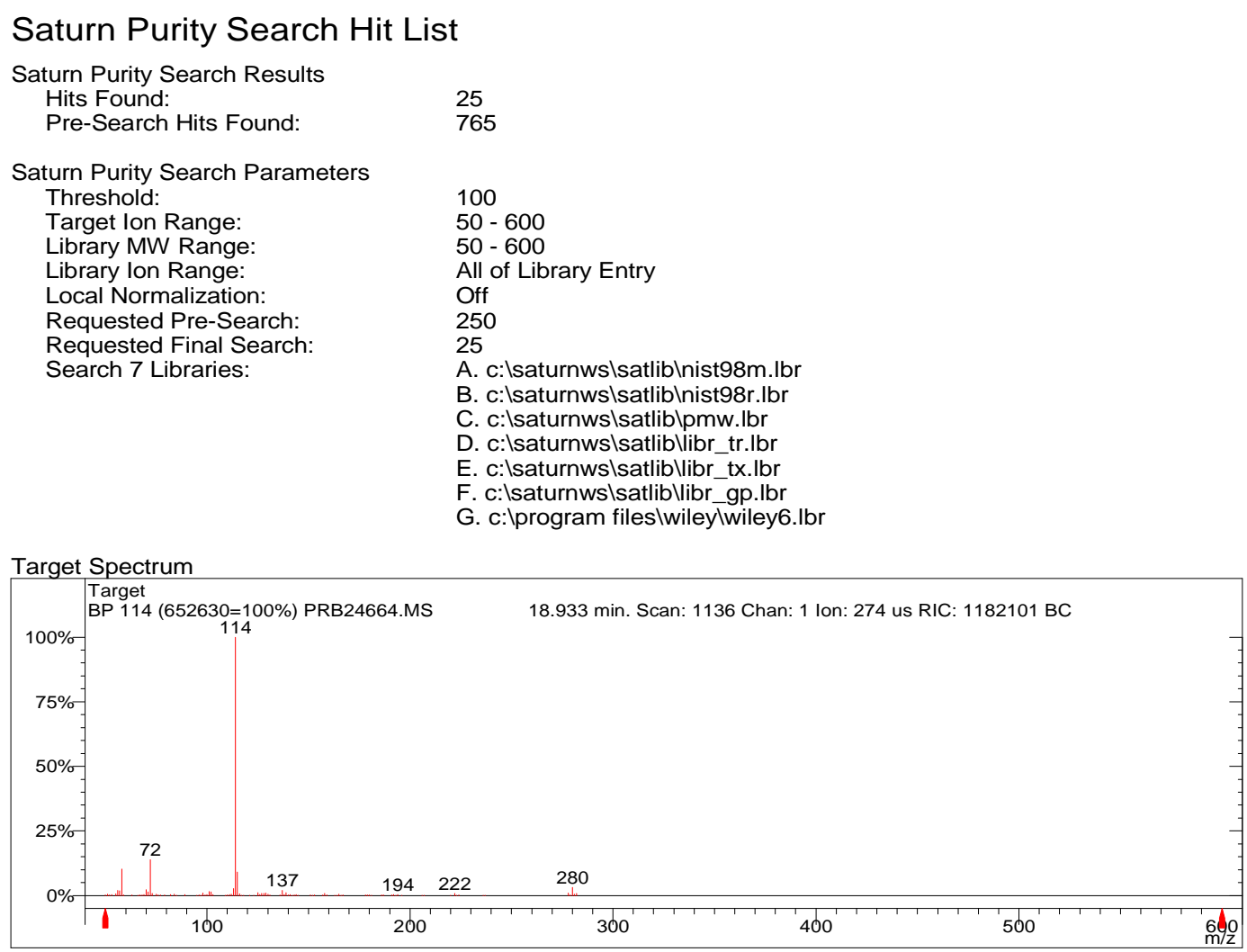

Spectrum from \ISaturn2000ไhdd saturn2\Saturn|DATAIPRB24664.MS

Scan No: 1136 , Time: 18.933 minutes

No averaging. Background corrected.

Comment: 18.933 min. Scan: 1136 Chan: 1 Ion: 274 us RIC: 1182101 BC

Pair Count: 99 MW: 0 Formula: None CAS No: None Acquired Range: 50 - 282

Purity Fit RFit Entry \# MW. Formula, CAS No., Name

$1925965938 \quad 8741 \mathrm{C} 280 \mathrm{C} 17 \mathrm{H} 26 \mathrm{ClN}, 106650-56-0$, Sibutramine

2. 69889075451423 A 380 C18H40N2S3, None, Ethane, 1-[(2-diisopropylamino)ethy]

366385076351413 A 517 C27H30Cl3N30, None, 1-[[2-[Butylamino]butyl]imino]-7-ch]

4. 63284470251422 A 114 C4H6N2S, 60-56-0, Methimazole

5. $6318297019084 \mathrm{G} 114 \mathrm{C} 4 \mathrm{H} 6 \mathrm{~N} 2 \mathrm{~S}$, 60-56-0, 2H-Imidazole-2-thione, 1,3-dihydro-1 6. $629761766 \quad 2305$ C 375 None, None,

7. $628 \quad 855 \quad 66534872$ G 159 C8H17NO2, None, N,N-dimethyl leucine $\$$

862573474451559 A 295 C21H29N, 5966-41-6, Benzenepropanamine, N, N-bis(1-methyl

961179370851416 A 157 C10H23N, 4458-33-7, Ethyl di-N-butylamine

D 61175867312442 B 129 C7H15NO, 13444-24-1, 3-Piperidinol, 1-ethyl-

11. 60384165951369 A 143 C8H17NO, None, Oxazolidine, 2,2-diethyl-3-methyl-

2 602850631121870 G 267 C11H26NO2PS, 71840-25-0, Phosphonothioic acid, methyl-,

B 60184963012428 B 267 C11H26NO2PS, 71840-25-0, Phosphonothioic acid, methyl-,

4. 59880066051419 A 233 C11H27NSSi, None, 2-Diisopropylaminoethanethiol, TMS C

\$ 58077660012432 B 129 C8H19N, 7087-68-5, Diisopropylethylamine

б 58077569251365 A 129 C8H19N, 16486-74-1, N-Butyl-tert-butylamine

1. 5797986669085 G 114 C4H6N2S, 60-56-0, 2H-Imidazole-2-thione, 1,3-dihydro-1

18. $577 \quad 717 \quad 779 \quad 2304 \mathrm{C} \quad 375$ None, None,

\$576 75170351409 A 129 C7H15NO, 57817-78-4, Oxazolidine, 3-ethyl-2, 2-dimethyl-

$20.57377069751404 \mathrm{~A} 228$ C14H32N2, None, 1,2-Bis-(2-diisopropylaminoethyl) et

$2572713631 \quad 18936 \mathrm{~A} 204$ C10H24N2O2, 26549-21-3, 1,4-Butanediamine, 2,3-dimethoxi

$2569740 \quad 663 \quad 51428$ A 267 C11H26NO2PS, 50782-69-9, VX

z 56373275151414 A 533 C28H31ClF3N3O2, None, 1-[[2-[Butylamino]butyl]imino]-7-c

Fig. 2. Sibutramine mass spectrum and the result of search in the mass spectra

\section{libraries.}


Scan 1151 from \ISaturn2000ไhdd saturn2\Saturn|DATAIPRB24665.MS

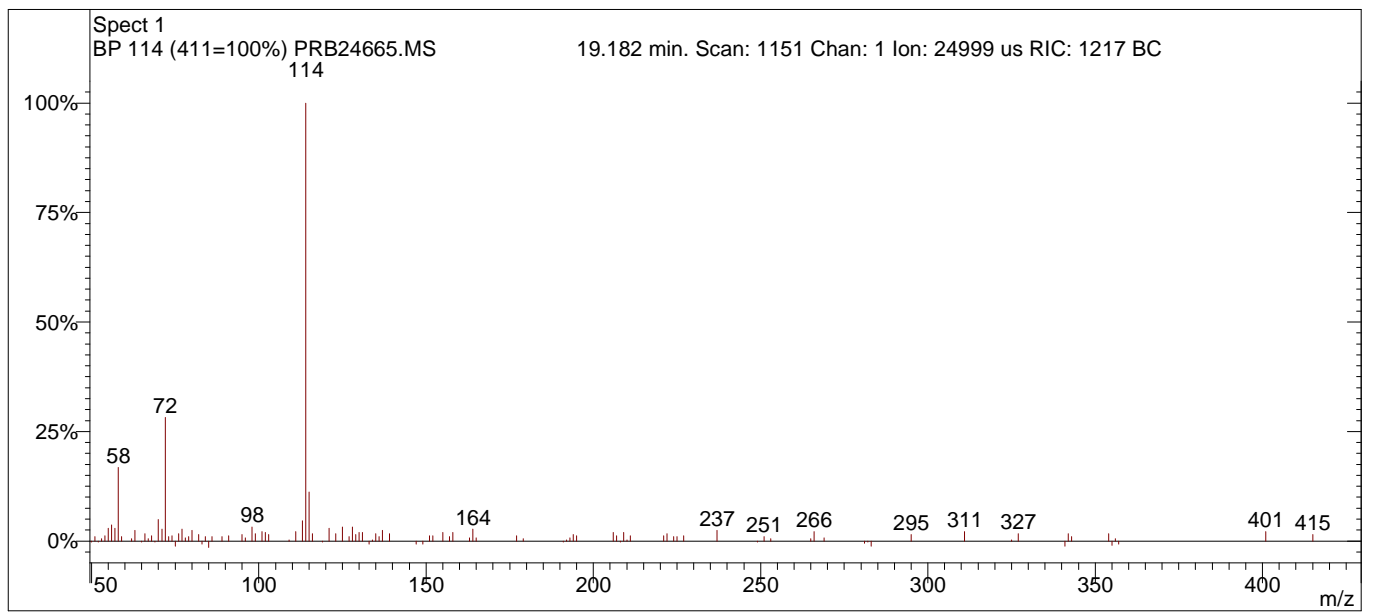

Spectrum from IISaturn2000/hdd saturn2ISaturn|DATAIPRB24665.MS

Scan No: 1151, Time: 19.182 minutes

No averaging. Background corrected.

Comment: 19.182 min. Scan: 1151 Chan: 1 Ion: 24999 us RIC: 1217 BC

Pair Count: 114 MW: 0 Formula: None CAS No: None Acquired Range: 50 - 429

\begin{tabular}{|c|c|c|c|c|c|c|c|c|c|c|c|}
\hline Ion & Int & $\div \mathrm{BP}$ & Ion & Int & $\because \mathrm{BP}$ & Ion & Int & $\div \mathrm{BP}$ & Ion & Int & $\because \mathrm{BP}$ \\
\hline 51 & 4 & 1 & 84 & 4 & 1 & 131 & 8 & 2 & 210 & 1 & 0 \\
\hline 53 & 2 & 0 & 86 & 4 & 1 & 134 & 1 & 0 & 211 & 5 & 1 \\
\hline 54 & 5 & 1 & 89 & 4 & 1 & 135 & 7 & 2 & 221 & 5 & 1 \\
\hline 55 & 12 & 3 & 91 & 5 & 1 & 136 & 4 & 1 & 222 & 7 & 2 \\
\hline 56 & 15 & 4 & 95 & 6 & 1 & 137 & 10 & 2 & 224 & 4 & 1 \\
\hline 57 & 12 & 3 & 96 & 3 & 1 & 139 & 7 & 2 & 225 & 4 & 1 \\
\hline 58 & 69 & 17 & 98 & 13 & 3 & 151 & 5 & 1 & 227 & 5 & 1 \\
\hline 59 & 4 & 1 & 99 & 7 & 2 & 152 & 5 & 1 & 237 & 10 & 2 \\
\hline 62 & 2 & 0 & 101 & 9 & 2 & 155 & 8 & 2 & 251 & 4 & 1 \\
\hline 63 & 10 & 2 & 102 & 8 & 2 & 157 & 4 & 1 & 253 & 2 & 0 \\
\hline 66 & 7 & 2 & 103 & 6 & 1 & 158 & 8 & 2 & 265 & 2 & 0 \\
\hline 67 & 2 & 0 & 109 & 1 & 0 & 163 & 3 & 1 & 266 & 9 & 2 \\
\hline 68 & 5 & 1 & 111 & 9 & 2 & 164 & 11 & 3 & 269 & 3 & 1 \\
\hline 70 & 20 & 5 & 113 & 19 & 5 & 165 & 3 & 1 & 295 & 6 & 1 \\
\hline 71 & 11 & 3 & 114 & 411 & 100 & 177 & 5 & 1 & 311 & 9 & 2 \\
\hline 72 & 116 & 28 & 115 & 46 & 11 & 179 & 2 & 0 & 325 & 1 & 0 \\
\hline 73 & 4 & 1 & 116 & 7 & 2 & 192 & 1 & 0 & 327 & 7 & 2 \\
\hline 74 & 5 & 1 & 121 & 12 & 3 & 193 & 3 & 1 & 342 & 7 & 2 \\
\hline 76 & 7 & 2 & 123 & 7 & 2 & 194 & 6 & 1 & 343 & 4 & 1 \\
\hline 77 & 11 & 3 & 125 & 13 & 3 & 195 & 5 & 1 & 354 & 7 & 2 \\
\hline 78 & 3 & 1 & 127 & 4 & 1 & 206 & 8 & 2 & 356 & 2 & 0 \\
\hline 79 & 4 & 1 & 128 & 13 & 3 & 207 & 5 & 1 & 401 & 9 & 2 \\
\hline 80 & 10 & 2 & 129 & 6 & 1 & 209 & 8 & 2 & 415 & 6 & 1 \\
\hline 82 & 6 & 1 & 130 & 8 & 2 & & & & & & \\
\hline
\end{tabular}

Fig. 3. Sibutramine mass spectrum at a concentration of $5 \mathrm{mg} / \mathrm{l}$.

\section{II.2.2 HPLC-DAD-MS Methods}

\section{II.2.2.1 HPLC-MS Method}

It is presented as a method for determining sibutramine in the injection matrices whose obtaining was described in paragraph 5.2.4.

Program duration: $30 \mathrm{~min}$.

ProStar solvent pumps

Constant flow $500 \mu \mathrm{l} / \mathrm{min}$ of solvent B

ProStar 410 Autosampler

Number of containers -84 of $2 \mathrm{ml} ; 3$ of $10 \mathrm{ml}$

Injection syringe volume $-250 \mu \mathrm{l}$

Injection loop volume $-100 \mu \mathrm{l}$ 
Volume hose connecting the needle for collecting the sample - $15 \mu 1$

Syringe speed $-1 \mu \mathrm{l} / \mathrm{s}$

Flush volume $-30 \mu \mathrm{l}$

Temperature of carousel with samples $40^{\circ} \mathrm{C}$

Use of sample bottle pressure

ProStar 500 column oven

Number of columns - four

Work Column 1 (C8-3)

Column temperature $-35^{0} \mathrm{C}$

Stabilization time $0.1 \mathrm{~min}$

Delays after transition $0.1 \mathrm{~min}$

1200 L mass spectrometer

ESI ionization type

Ionization type - positive

Scan speed $1 \mathrm{scan} / \mathrm{s}$

Detector voltage $-1480 \mathrm{~V}$

Peak width of the first quadrupole $-0.7 \mathrm{amu}$

Peak width of the third quadrupole $-0.9 \mathrm{amu}$

Scan range $50-350 \mathrm{amu}$

Detector ProStar with diode area 335

Acquisition range - 200 to $400 \mathrm{~nm}$

Slit width $-2 \mathrm{~nm}$

Minimum purity level $-220 \mathrm{~nm}$

Maximum purity level - $360 \mathrm{~nm}$

Absorption spectrum acquisition: for each $\mathrm{nm}$ from the acquisition field Display of absorption variation by two wavelenghts: 254 and $280 \mathrm{~nm}$

Noise monitoring by 26 points.

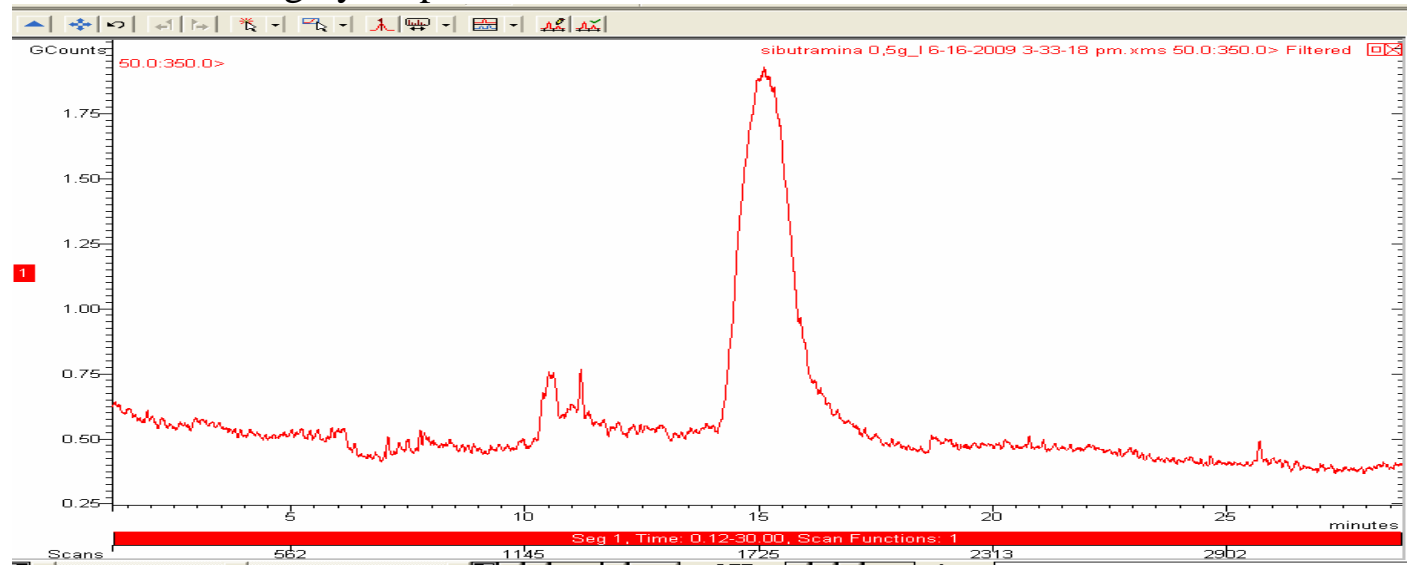

Fig. 4, Total ion chromatogram of sibutramine at $0.5 \mathrm{~g} / \mathrm{l}$. 


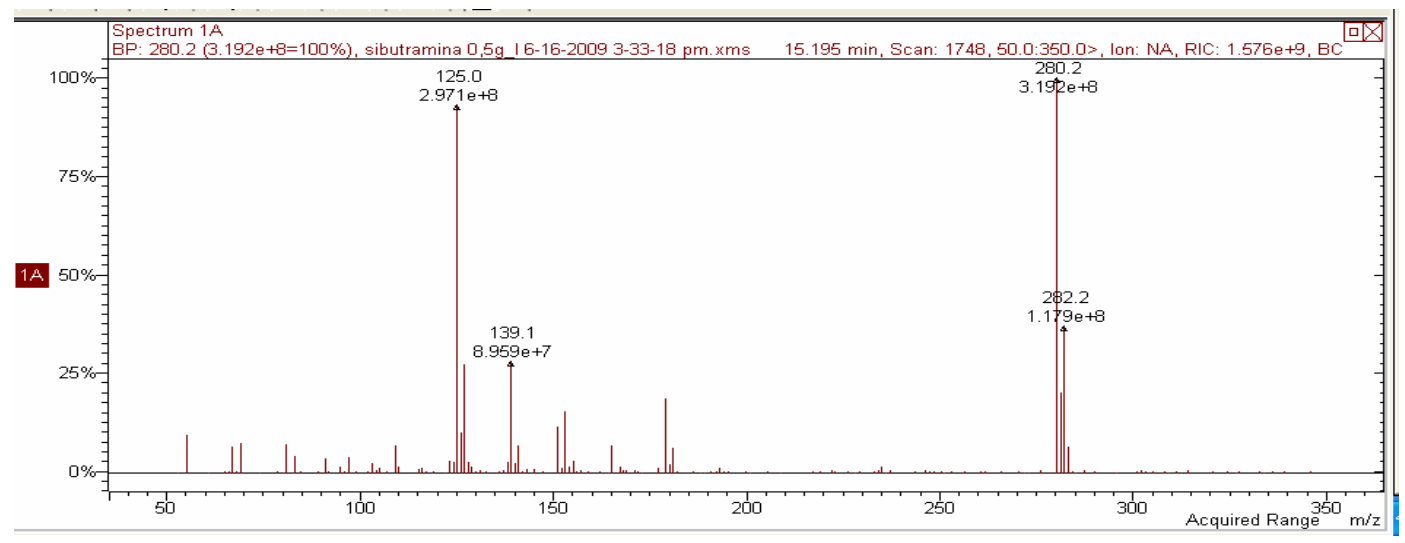

Fig. 5. Sibutramine mass spectrum at $0.5 \mathrm{~g} / \mathrm{l}$.

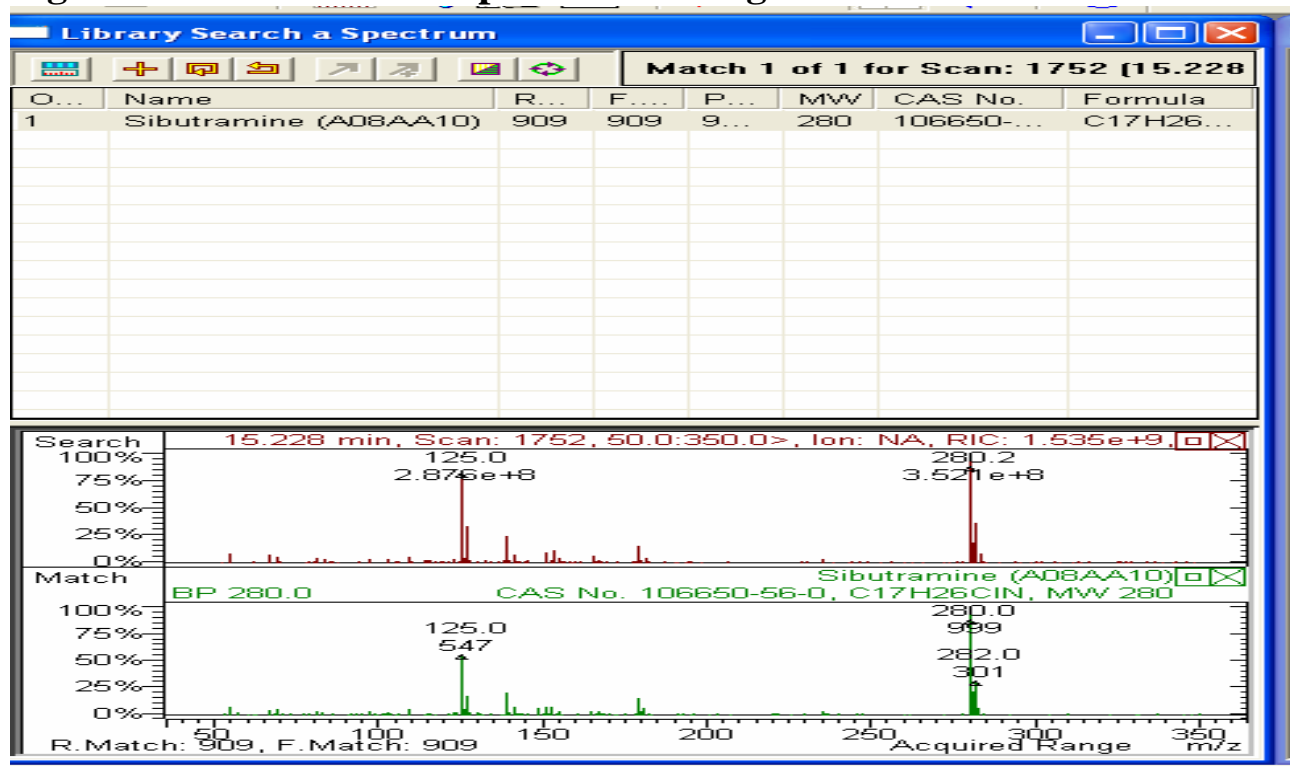

Fig. 6. Mass sibutramine spectrum at $0.5 \mathrm{~g} / \mathrm{l}$ as compared with that found in the MI spectra library of the HPLC-MS system.




Fig. 7. Total ion chromatogram of sibutramine in a matrix of three solvents compared to a liquid chromatogram obtained at $254 \mathrm{~nm}$ (black) and $280 \mathrm{~nm}$ (red).

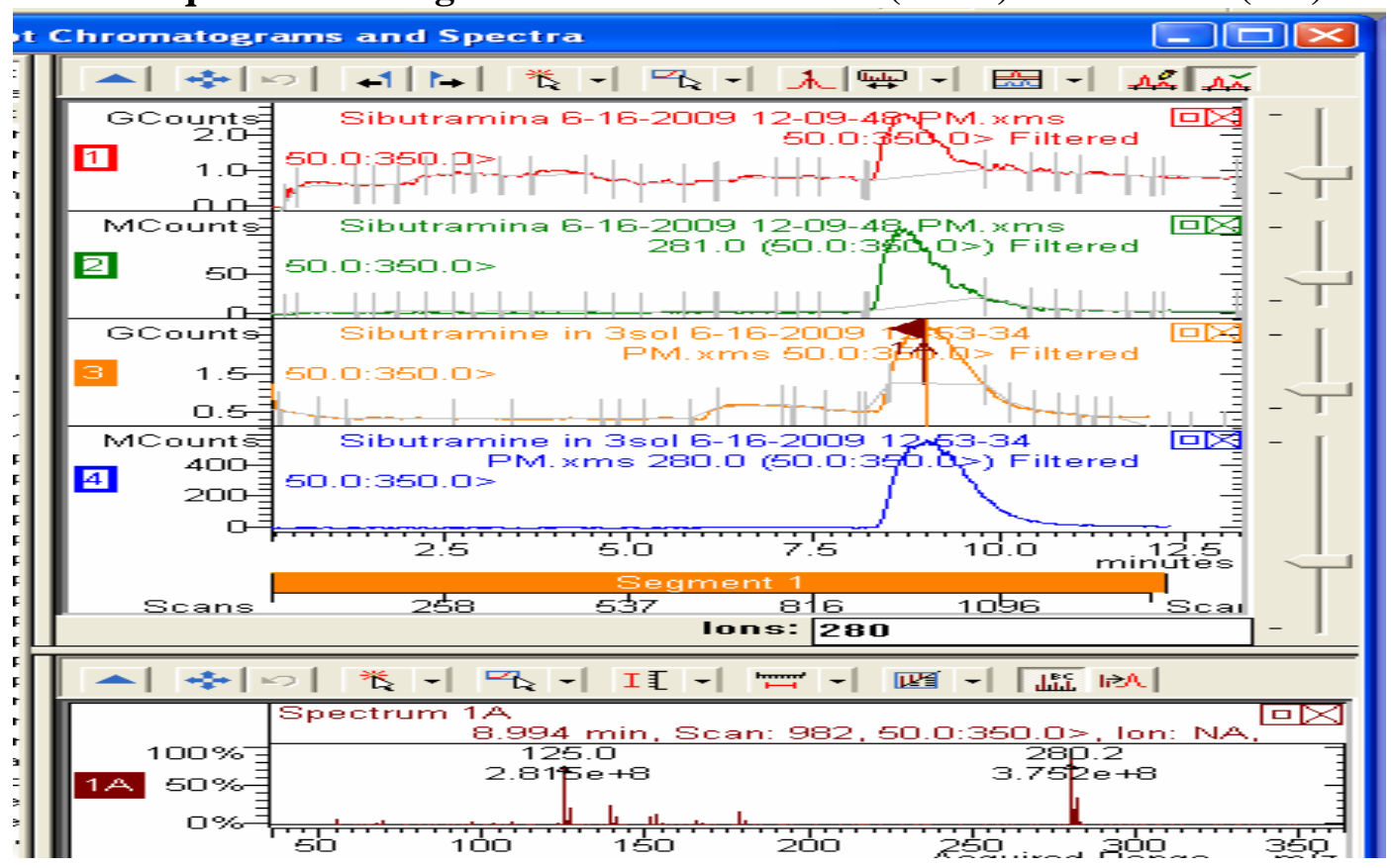

Fig. 8. Total ion chromatograms (tracks 1 and 3) and ion chromatograms 281 (routes 2 and 4) of sibutramine at $0.5 \mathrm{~g} / \mathrm{l}$ in methanol + acetonitrile matrix (tracks 1 and 2) and three solvent matrix (tracks 3 and 4 ).

\section{II, 2.2. HPLC MS/MS Method}

It is presented as a method for determining sibutramine through the MS/MS technique.

Program duration: 30 min.

ProStar solvent pumps

Constant flow $500 \mu \mathrm{l} / \mathrm{min}$ of solvent B

ProStar 410 Autosampler

Number of containers -84 of $2 \mathrm{ml} ; 3$ of $10 \mathrm{ml}$

Injection syringe volume $-250 \mu \mathrm{l}$

Injection loop volume - $100 \mu \mathrm{l}$

Volume hose connecting the needle for collecting the sample $-15 \mu \mathrm{l}$

Syringe speed $-1 \mu \mathrm{l} / \mathrm{s}$

Flush volume $-30 \mu \mathrm{l}$

Temperature of carousel with samples $40^{\circ} \mathrm{C}$

Use of sample bottle pressure

ProStar 500 column oven

Number of columns - four

Work Column 1 (C8-3)

Column temperature $-35^{0} \mathrm{C}$

Stabilization time $0.1 \mathrm{~min}$

Delays after transition $0.1 \mathrm{~min}$

1200L mass spectrometer

ESI ionization type

Ionization type - positive 
Scan speed $1 \mathrm{scan} / \mathrm{s}$

Detector voltage - $1480 \mathrm{~V}$

Peak width of the first quadrupole - given by calibration

Peak width of the third quadrupole - given by calibration

Step $1 \mathrm{Q}_{1}-280 \mathrm{amu} ; \mathrm{Q}_{3}-97 \mathrm{amu}$; with collision voltage: $-10.0 \mathrm{~V}$

Step $2 \mathrm{Q}_{1}-280 \mathrm{amu} ; \mathrm{Q}_{3}-139 \mathrm{amu}$; with collision voltage: $-11.5 \mathrm{~V}$

Step $3 \mathrm{Q}_{1}-280 \mathrm{amu} ; \mathrm{Q}_{3}-153 \mathrm{amu}$; with collision voltage: $-11.0 \mathrm{~V}$.

Detector ProStar with diode area 335

Acquisition range - 200 to $400 \mathrm{~nm}$

Slit width $-2 \mathrm{~nm}$

Minimum purity level $-220 \mathrm{~nm}$

Maximum purity level - $360 \mathrm{~nm}$

Absorption spectrum acquisition: every $2 \mathrm{~nm}$

Display of absorption variation by two wavelenghts: 254 and $280 \mathrm{~nm}$

Noise monitoring by 27 points.
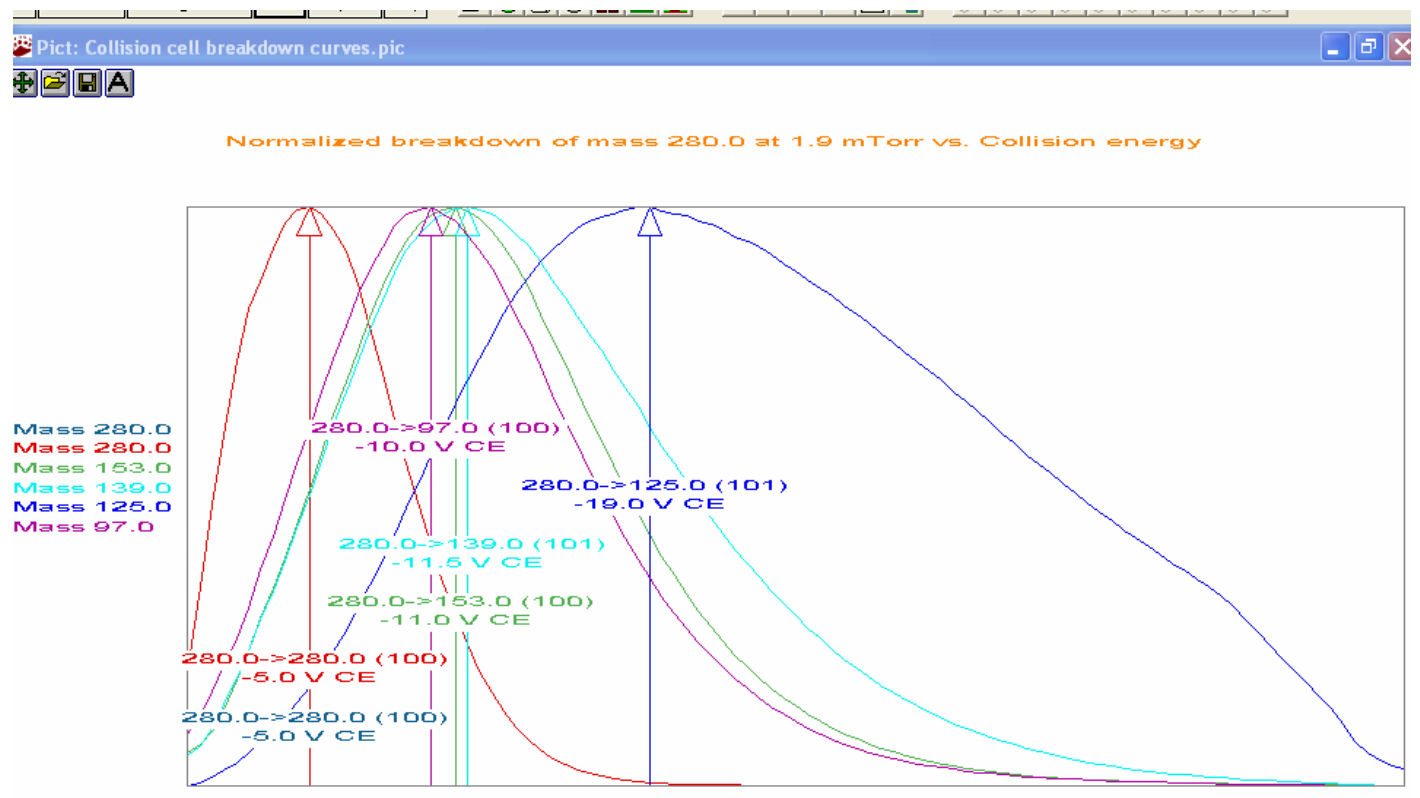

itrl

Fig. 9. Result of ion $\mathbf{2 8 0}$ dissociation selected from the mass spectrum of sibutramine. 




Fig. 10. The MS/MS spectrum of sibutramine in a concentration of $5 \mathrm{mg} / \mathrm{l}$.



Fig. 11. Comparison between the MS/MS spectrum of sibutramine in concentration of $5 \mathrm{mg} / \mathrm{l}$ (tracks 1-3) and that obtained at a concentration of $0.5 \mathrm{mg} / \mathrm{l}$. 


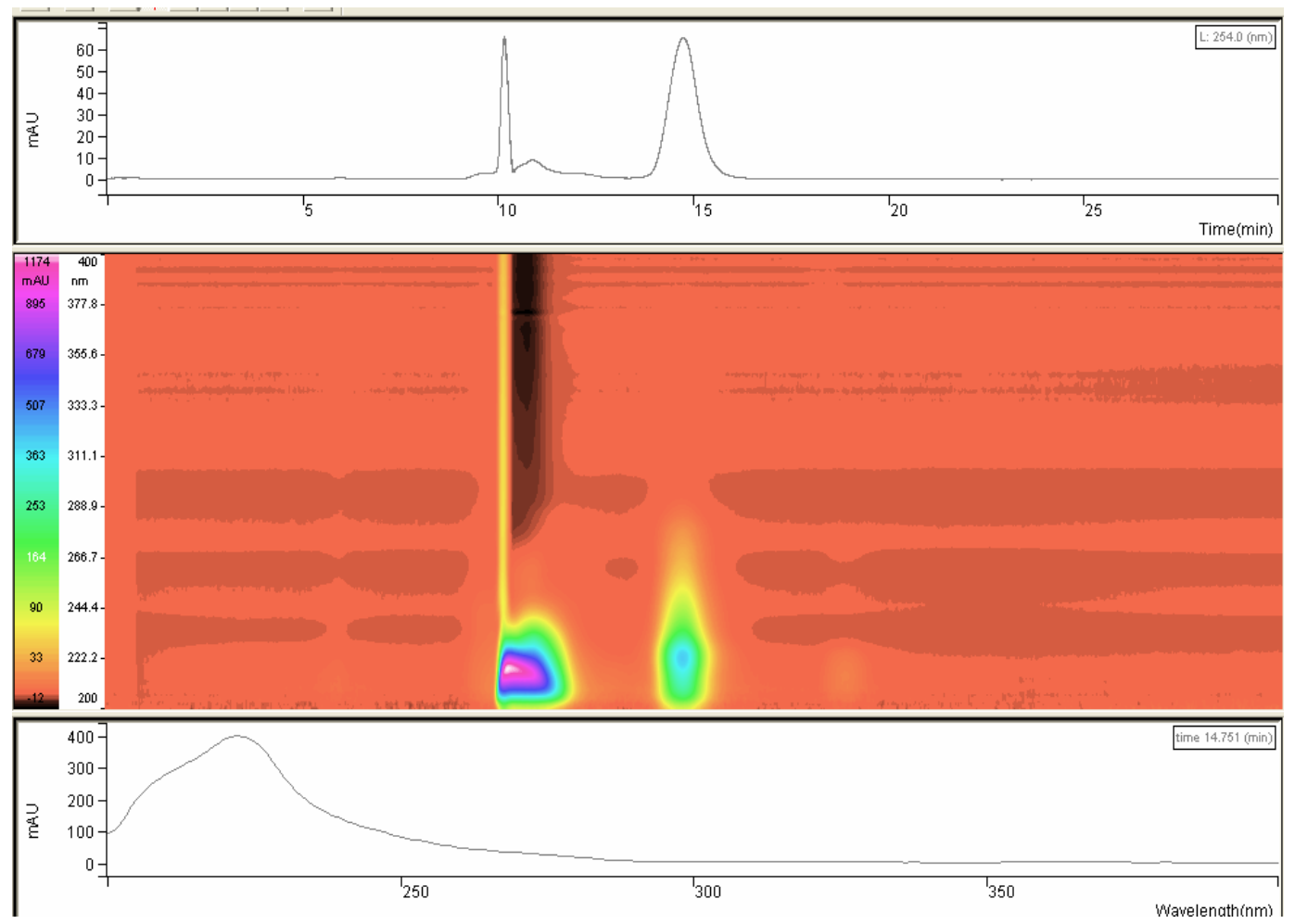

Fig. 12. Liquid chromatocartogram of sibutramine in concentration of $0.5 \mathrm{~g} / \mathrm{l}$.

\section{DISCUSSION}

\section{IV.1. Pharmacology}

IV.1.1. Pharmacological effects

Another characteristic effect of sibutramine is its anorexigenic nature. Amphetamine decreases appetite and facilitates compliance with hypocaloric diet by the obese, helping weight loss.

The effects on the central nervous system are due to their interference at the level of catecholaminergic synapses. Amphetamine releases catecholamines from presynaptic terminals, reduces their uptake in these endings and inhibits their intraneuronal inactivation by monoamine oxidase. Psychomotor stimulation corresponds to an increase in the activity of the ascending reticular activating system, favouring the attentiveness process. Thus, wakeness reactions are amplified and there is a state of alertness in connection with the elective action on noradrenergic neurons, with the release of noradrenaline, with certain central synapses. The anorexigenic effect is due to the hypothalamic feeding center and corresponds to a local release of noradrenaline and dopamine. Stimulation of motility and motor stereotypes, arising from high doses, are assigned to dopamine release in the striated muscle. Psychotic phenomena produced by toxic doses are due to the release of dopamine in the mesolimbic system and to the release of serotonin.

Sibutramine also has sympathomimetic effects, i.e. there is an increase in blood pressure, weak bronchodilation, sphincter contraction and relaxation of the bladder fundus, increase of fatty acid concentration in the plasma.

In patients with narcolepsy sibutramine allows sleep seizure control without changing the state of catalepsy. Its therapeutic benefit lies in delaying in the development of fast sleep.

In the case of hyperkinetic syndrome in children (ADHD), sibutramine is effective especially in school children. The drug produces a decrease in the state of anxiety, motor agitation. The capacity for attention also increases without diminishing the learning process and mitigatess at least in part impulsiveness and behavioural disturbances. 
In Parkinson's, the therapeutic benefits consist in reducing rigidity, oculogyric crisis prevention, improved mood and better sleep.

In epilepsy, it has a certain special efficacy regarding Petit Mall crises and antagonizes central depressant unwanted effects.

Amphetamine is readily absorbed in the intestine, being effective if taken orally. It has a half-life of 7-14 hours. It is eliminated through urine, and its metabolism is via the liver. In acidic urine a large quantity of amphetamine is released, as the $\mathrm{pH}$ decrease - increases the proportion of the dissociated form.

\section{IV.1.2. Dose administration}

Amphetamine use is limited due to its aggressive neuro-central effects. Its psychomotor stimulant effect recommands it for use only in special circumstances that require mandatory increased psychomotor performance and the removal of the feeling of fatigue.

Its dosage is in amounts of 3-6 mg/day 2-3 times a day, the last dose being administered before 4 p.m., to avoid insomnia at night. The usual dose in humans causes mental excitation phenomena with feelings of freshness, fun, initiative, increased concentration, the need to talk, the appearance of fatigue being delayed.

Thus, this "state of well-being" leads to developing tolerance, to phenomena of chronic intoxication, psychiatric disorders and a state of addiction, involving a change from the condition of taking a medicine to that of drug consumer, a background that favours the consumption of other substances with high toxicity as well.

\section{IV.2 Toxicology}

Tolerance initially concerns peripheral, sympathomimetic effects, which quickly become progressively weaker and which include a number of effects on nerves + psychomotor stimulation, euphoria, anorexia - and the lethal effect (a person may survive doses of $500 \mathrm{mg}$ to 1 gram, when exitus occurs). Development of tolerance requires progressively higher doses that produce chronic neurotoxic and psychotoxic phenomena hyperactivity, irritability, tremors, motor stereotypes, mental disorder with delusions and hallucinations similar to those in paranoid schizophrenia.

\section{Bibliography}

Yamamoto S-Shokuhin Eiseigaku Zasshi, 369, 2011

L. Enescu - Drug syntheses and uses

Bray G A - Use and abuse of appetite - suppresant drugs in the tratament of $\underline{4 . F o o d}$ and Drug AdministrationNovember 241997FDA APPROVES SIBUTRAMINE TO TREAT $\underline{O B E S I T Y^{\wedge}}$

Bruce Japsen (13 March 2005). "FDA weighs decision on Meridia; Health advisory likely for Abbott obesity drug". Chicago Tribune. Chicago, Illinois;

Miranda-G E, Sordo M, Salazar AM, et al. (2007). "Determination of amphetamine, methamphetamine, and hydroxyamphetamine derivatives in urine by gas chromatography-mass spectrometry and its relation to CYP2D6 phenotype of drug users". J Anal Toxicol 31 (1): 31-6. PMID 17389081.

Amphetamine-Induced Dopamine Release in Human Ventral Striatum Correlates with Euphoria. Psychiatry

Clement, Beverly A., Goff, Christina M. and Forbes, T. David A. (1998). Toxic amines and alkaloids from Acacia rigidula. Phytochemistry

Ionică M. The HPLC MS/MS Varian System - professional training course 